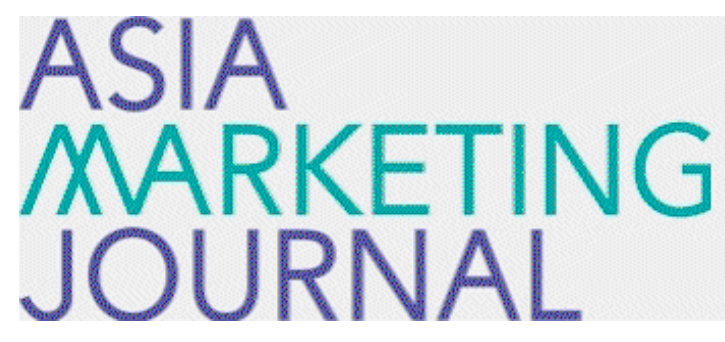

ASIA MARKETING JOURNAL

Volume 21 | Issue 4

Article 1

$1-31-2020$

\title{
What Brings Customer Gapjil? The Intertwined Effects of Perceived Economic Mobility, Self-Other Referent Priming, and Temporal Focus
}

Yongju Kwon

Youjae Yi

Follow this and additional works at: https://amj.kma.re.kr/journal

Part of the Marketing Commons

\section{Recommended Citation}

Kwon, Yongju and Yi, Youjae (2020) "What Brings Customer Gapjil? The Intertwined Effects of Perceived Economic Mobility, Self-Other Referent Priming, and Temporal Focus," Asia Marketing Journal: Vol. 21 : Iss. 4 , Article 1.

Available at: https://doi.org/10.15830/amj.2020.21.4.1

This Article is brought to you for free and open access by Asia Marketing Journal. It has been accepted for inclusion in Asia Marketing Journal by an authorized editor of Asia Marketing Journal. 


\title{
What Brings Customer Gapjil? The Intertwined Effects of Perceived Economic Mobility, Self-Other Referent Priming, and Temporal Focus*
}

\author{
Yongju Kwon** \\ Youjae $\mathrm{Yi}^{* * *}$
}

\begin{abstract}
The current research brings the spotlight onto customer Gapjil toward service employees. In an attempt to understand what brings Gapjil, the present article investigates the intertwined effects of perceived economic mobility (PEM), self-other referent priming (SORP), and temporal focus on Gapjil. Study 1 shows that PEM increases Gapjil among self-referent primed people, but not among other-referent primed people. Study 2 examining the role of temporal focus (present vs. future) reveals that the effect found in study 1 is replicated in the present focus, but the effect is reversed in the future focus. We explain this dynamic pattern of the 3-way interaction effect with a relative gratification and a motivation to attune the self to the perceived norm of the high class. We also discuss how to create a social atmosphere to lessen Gapjil in public and corporate communication strategies.
\end{abstract}

Keywords: Gapjil, perceived economic mobility, self-other referent priming, temporal focus, relative gratification

\section{Introduction}

A few years ago, an executive of a big Korean conglomerate assaulted and hit a female flight attendant with a magazine for serving undercooked ramen. More recently, a mother and a daughter who visited a department store made parking attendants kneel down, because they were upset when the attendants asked to

This research is supported by the Institute of Management Research, Seoul National University.

** Doctoral student, College of Business Administration, Seoul National University (shakes52@snu.ac.kr)

*** Professor of marketing, College of Business Administration, Seoul National University (youjae@snu.ac.kr),

Corresponding Author 
move their car which was parked at a space for two. A number of media reported these cases with the word 'Gapjil,' and it has attracted a tremendous amount of attention among people. According to BIG Kinds, a website for news search service by Korea Press Foundation, the number of news articles including the keyword 'Gapjil' has dramatically increased from 5 in 2012 to 18,013 in 2018 (Jeong and Jo 2019). Google search index also indicates that more and more citizens have searched 'Gapjil' in google, as the index has increased from 1 in 2012 to 607 in 2018.

According to a survey on perceptions of Gapjil and Gap-Eul relationships, 96\% of participants indicated that Gapjil has reached a serious level in our society, and 90\% experienced
Gapjil in their daily lives (Jeong and Jo 2019). In addition, 85\% of participants evaluated that Gapjil in the relationship between emotional laborers and customers has reached a serious level, and 57\% perceived "customers" as a group of people conducting Gapjil. In an essay titled “Memories of Gapjil” (Seo 2015), the author describes a conversation with a neighbor who used to own an outdoor clothing store. The store owner went out of business due to stress from customers returning clothes after several days, claiming that they had never worn clothes. However, clothes were stained with kimchi liquid and smelled grilled pork belly. Indeed, Gapjil conducted by customers is pervasive in reality. When the National Police Agency enforced a special crackdown on Gapjil,

〈Figure 1〉 Gapjil Search Trend

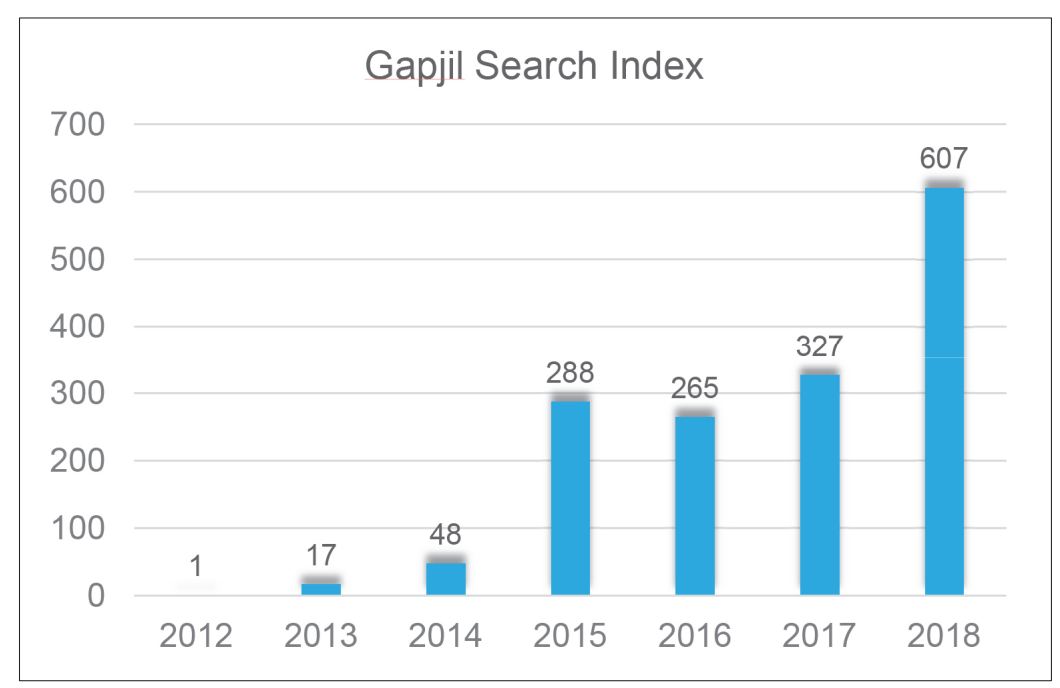

Reference: trends.google.com

Index of each year $=$ Sum of search index of each month during the year

The range of index (each month) $=0 \sim 100$ 
$43.7 \%$ of 7,633 people arrested were black or dysfunctional consumers (Kim 2016.12.15).

Although media and citizens are paying more and more attention to Gapjil, academic efforts have relatively lagged behind. Only a few previous works in literatures on social science (Choi 2018), law (Park 2016), political science (Jeong and Jo 2019), psychology (Lee 2016b), pastoral theology (Lee 2016a), and hotel management (Cho 2018; Ryu and Ryu 2017) have examined Gapjil. This paucity of academic research is particularly true in the marketing field. When the keyword 'Gapjil' was searched among articles published in the Asia Marketing Journal, Korean Journal of Marketing, and Journal of Consumer Studies, only one article (Jeon and You 2014) published in KJM just mentioned the keyword in an abstract without any further investigation. Overall, none of papers published in three major marketing journals in Korea has investigated Gapjil. This result is astonishing, considering the intensive media coverage of customer Gapjil and its severe adverse effects on service employees and emotional laborers. More importantly, few papers published in journals of other academic fields have empirically tested antecedents of Gapjil. This limitation in academia is crucial in that figuring out what brings Gapjil is the first step to find solutions for reducing Gapjil.

To close the gap, the present research aims to empirically investigate what brings customer Gapjil. More specifically, the current article investigates how perceived economic mobility (henceforth PEM) affects customer Gapjil depending on whether people think about themselves or others (i.e., self-other referent priming). In addition, the current research investigates the role of temporal focus moderating the interaction effect of PEM and self-other referent priming on customer Gapjil. By doing so, the present research shows that optimistic perception of social structure affects customer Gapjil, and this effect dynamically varies according to which people are temporally and intra- or inter-personally focused on.

\section{Theoretical Background}

\section{1 (Customer) Gapjil}

The first and second words of the ten Heavenly Stems (i.e., Shipgan in Korean, which is a collective term of Gap, Eul, Byung, Jung, Mu, Gi, Gyung, Shin, Im, and Gye) 'Gap' and 'Eul' - are normally used to describe the relationship between superiors (i.e., Gap) and subordinates (i.e., Eul). The word 'Gapjil' is a newly-coined word which is a combination of 'Gap' referring to superiors holding power and 'jil' meaning misbehaviors. Although Gapjil is a slang that is frequently used but not defined clearly, most members in the Korean society generally understand and share the 
concept of Gapjil (Yoon et al. 2018). Based on previous studies, Gapjil is defined as wrongful words and actions of Gap - person(s) or groups that are superior to Eul in terms of authority, power, or status - which inflict harm upon Eul physically, psychologically, emotionally, or materially for unjustified benefits or satisfaction of 'Gap' (Jeong and Jo 2019; Lee 2016a; Ryu and Ryu 2017). Gapjil occurs under three conditions: 1) there is power asymmetry between Gap and Eul, 2) Eul has a high level of dependence on Gap, and 3) Eul is compelled to accept arbitrary interference by Gap in order to maintain one's fundamental benefits (Jeong and Jo 2019).

Even though Gapjil has been perceived as misbehaviors of Chaebols or the top rank officers toward ordinary citizens, Gapjil has become more widespread throughout the whole community (Choi 2018). Even ordinary people are doing Gapjil toward other ordinary people who are seemingly inferior. For example, customers are typically perceived as 'Gap' in the relationship between customers and service employees; many firms emphasize that the customer is the king. In the present article, customer Gapjil refers to wrongful words and actions of customers - persons or groups that are potentially influential in sales of products or services, word of mouth, and pressure by reporting complaints - which are beyond common sense and inflict harm upon service employees, emotional laborers, stores, or companies physically, psychologically, emotionally, or materially for unjustified benefits or satisfaction of customers (Cho 2018; Lee 2016a). Henceforth, Gapjil in this article will mostly indicate 'customer Gapjil' for convenience.

Previous studies have mostly focused on the negative consequences of Gapjil and found that Gapjil increases tension, anxiety, emotional burnout, turnover intention, dissatisfaction, and helplessness but decreases service recovery efforts, performance, subjective life satisfaction, and self-esteem of service workers (Gong 2015; Jeong and Jo 2019; Moon 2018; Ryu and Ryu 2017; Yi 2014). The detrimental effects of Gapjil are not confined to service workers or emotional laborers. Gapjil has also negative influences on service companies in terms of loss of trust in the system, organizational helplessness, and lowered sales volume and quality of sales interaction (Enosh et al. 2013; Rafaeli et al. 2012; Verbeke and Bagozzi 2002). Aforementioned works examined adverse consequences of customer Gapjil for service workers and companies. However, in order to prevent Gapjil, it is important to understand what brings Gapjil.

Considering the lack of previous studies on customer Gapjil in the marketing literature, we extended literature review to other academic fields and found that a few studies have suggested some possible causes of Gapjil in a general context. Lee (2016a) proposed the unfair relational morals offered in Confucianism, which favor the stronger, are one of reasons for the prevailing Gapjil phenomenon. He also proposed 
a less-developed psychological structure of superordinate people as another possible reason. Pathological narcissism was also examined as an antecedent of Gapjil (Lee 2016b). Choi (2018) viewed that widespread Gapjil among ordinary people is rooted from relative deprivation, the desire to become superior to others, the need to survive in a highly competitive society, the intensification of emotional labor, and the level of social trust in the Korean Society. Although these studies offer propositions which might be helpful in guiding the directions of future research, none of them empirically tested the relationships between proposed causes and Gapjil.

In summary, most empirical studies on this topic examined the negative consequences of Gapjil. Previous works regarding the antecedents of Gapjil only provided propositions, which were not empirically tested. Considering this gap, the current research not only proposes PEM, self-other related thoughts (i.e., self-other referent priming), and temporal focus as antecedents of Gapjil but also tests their intertwined effects on Gapjil. By empirically testing this dynamic relationship, the present research also draws practical remedy for Gapjil.

\subsection{PEM and Customer Gapjil}

A recent survey in South Korea revealed that most respondents $(90.3 \%)$ of the survey recognized so-called "Spoon Hierarchical Theory"
(Han 2018). The lay theory reflects desperate beliefs that a child's life is pre-determined by the type of spoons (i.e., gold, silver, bronze, or dust) given to a child at birth depending on parents' wealth. Perceived economic mobility (PEM) is highly related with the lay theory. PEM is defined as a personal belief about the extent to which one's society allows its members to climb up the economic ladder (Yoon and Kim 2016). To be more specific, if people believe that everyone can be rich regardless of the type of spoons given at birth, they have high PEM. Conversely, low PEM reflects the belief that children with dust spoons cannot reach a higher economic status even if they make a lot of efforts.

Then, how does PEM affect Gapjil? Some hints exist in the sociology literature. Dambrun et al. (2006) found that potential recipients of future gratification show greater oppression toward the immigrant out-group. Guimond and Dambrun (2002) also warned that people who expect an enhanced status in the future display more prejudice. Findings of Postmes and Smith (2009) provided a possible explanation about aforementioned studies that people who expect to achieve a privileged position in the future are more likely to adjust their behaviors, intentions, or expressions to the norms of their prospective in-group for the purpose of displaying good citizenship. Lack of rich role models fulfilling noblesse oblige and frequent media exposure of disrespectful aspects of the rich 
within a society might make people perceive incivility or Gapjil as a norm of the high class. Considering that people with high (vs. low) PEM believe to move up the economic ladder and receive future gratification, they are more likely to attune their words and actions to the perceived norm of the high class than are those with low PEM. Indeed, Kwon and Yi (2019) found that PEM increases customer aggression toward service employees, an operationalization of Gapjil.

Will PEM increase Gapjil under any circumstance? The current research posits that the effect of PEM on Gapjil varies depending on the relative attention people give to themselves vs. others. In the next section, we will examine the potential role of self-other referent priming and propose a relevant hypothesis.

\subsection{The Moderating Role of Self-Other Referent Priming}

Previous research has shown that a situational activation of a concept affects reactions in later, unrelated situations (Förster et al. 2007). Similarly, self-other referent priming (hereafter SORP) can also have an influence on later judgments or choices. SORP affects the relative attention people give situationally to either themselves or others and thus increases the accessibility of concepts associated with different targets (Fenigstein and Levine 1984; Wan et al. 2011).
With respect to the memory of concepts, self-referent knowledge and other-referent knowledge are differently encoded and have different structures (Krueger and Stanke 2001). To be more specific, the self is the center of consciousness (Wood and Cowan 1995), and self-referent knowledge is constructed based on the sense of self which has an inextricable connection with direct phenomenal experiences (Krueger and Stanke 2001). The sense of self is not limited to the past or the present moment in time. Rather, it extends to the future as well (Peetz and Wilson 2014). Compared with other-referent knowledge, self-referent knowledge is more deeply encoded, more highly structured and more easily accessible (Krueger and Stanke 2001). Accordingly, when people give the relative attention to the self, they might easily access to self-evaluation which is a highly structured sense of self interwoven with the accumulation of direct phenomenal experiences of the past, present, and future.

In terms of social status and power, people also have the structured sense of self based on their direct experiences of education and socioeconomic activities such as work. PEM is likely to make a difference in the following manner. People with high PEM might form the sense of self by considering even their future potentialities of being rich in addition to their accumulated direct experiences of the past and the present, whereas those with low PEM might not do so. Rather, people with low 
PEM might constitute the sense of self either by considering accumulated direct experiences of the past and the present only or by adding a pessimistic imagination about self-status in the future. Overall, among self-referent primed people, those with high (vs. low) PEM are more likely to perceive relative gratification by considering potentialities in self-evaluation, attune their words and actions to the perceived norm of the potential in-group (i.e., the rich), and thus do Gapjil toward service employees.

However, when people give the relative attention to others, they cannot help taking other-referent knowledge into account in selfevaluation. Given that self-referent knowledge is almost always present (Krueger and Stanke 2001), high accessibility of other-referent knowledge might activate social comparison between the self and others. Unlike self-referent knowledge, other-referent knowledge is mainly composed of inferences based on observations of others' words and deeds (Krueger and Stanke 2001). Overall, when people give the relative attention to others, they might form otherreferent knowledge by observing currently vivid, actual words and actions of others. In response to this 'vivid' other, the self, as the corresponding target of social comparison, might be composed of more vivid experiences just as he or she is. In this case, potentiality such as PEM hardly affects the sense of self, because it is just a hopeful belief that is not actualized yet here and now. Thus, when people are other-referent primed, PEM is less likely to affect the perception of relative gratification and Gapjil. Therefore, the first hypothesis is proposed as follows:

Hypothesis 1: PEM will increase customer Gapjil toward service employees when people are self-referent primed, whereas PEM will not when people are other-referent primed.

\section{Study 1: The Interaction Effect of PEM and SORP on Gapjil}

The goal of study 1 is to investigate how the effect of PEM on customer Gapjil varies depending on SORP in an online experiment setting. Both PEM and SORP are situationally manipulated in this study. By measuring customer Gapjil toward service employees following the manipulation tasks, the current study aims to test how PEM, SORP, and their interaction causally affect Gapjil. PEM is expected to increase Gapjil when people are thinking about self (self-referent primed) but to have little effect when they are thinking about others (other-referent primed).

\subsection{Method}

A total of 245 participants were recruited 
from Prolific, which is a British online subject pool company. Among those participants, fourteen people who failed in attention checks or a PEM article summary task were excluded in data analysis (Oppenheimer et al. 2009). As a result, 231 people (71 males, 159 females, 1 other, mean age $=37.6$ ) were included in our analysis. Participants were composed of 91.3\% Whites, 3.9\% Asian/Asian British, 2.2\% Mixed/ Multiple ethnic groups, 1.7\% Black/Black British/African/Caribbean, and .9\% other. All were British citizens. The present study was designed as a 2(PEM: low vs. high) x 2(Selfother referent priming: self vs. other) betweensubjects experiment.

\subsubsection{PEM}

Participants were randomly assigned to an either high or low PEM condition and read a bogus article. The way of PEM manipulation was adapted from previous work (Yoon and Kim 2016). We modified the US-based original stimuli for British participants such that all American celebrities, media sources, institutions, pop cultures mentioned within the stimuli were replaced with corresponding British ones (see Appendix). In the high PEM condition, participants read an article arguing that the U.K. is a land of high economic mobility (e.g., "19 percent of British men raised in the bottom fifth of incomes rose to the top fifth. Meanwhile, just 11 percent of British men at the bottom stay there as adults"). In the low PEM condition, participants read an article describing the U.K. as a country of low PEM (e.g., "67 percent of British men raised in the bottom fifth of incomes stay there as adults. Meanwhile, just 4 percent of British men at the bottom rose to the top fifth"). To enhance participants' comprehension of the article, the current study included additional tasks such as clicking four to five key sentences of the article and summarizing the article with a couple of sentences. After the tasks, participants answered two-item bipolar manipulation check questions of PEM (e.g., "my future economic status mainly depends on what I am given at birth" = 0 to "my future economic status mainly depends on what I do today" = 10 , adopted from Yoon and Kim 2016, $a=.75$ ).

\subsubsection{SORP}

After PEM-related tasks, participants were randomly assigned to an either self-referent or other-referent priming condition adopted from Wan et al. (2011). In the self-referent priming condition, participants were asked to write ten sentences which best describe themselves ("please describe yourself by writing 10 statements starting with 'I am"'). To make sure that participants focus on themselves not others, additional instructions were included ( "you should type 'I am' at the beginning of every sentence"). In the other-referent priming 
condition, participants were requested to write ten sentences which best describe one of their friends ("please describe your friend by writing 10 statements starting with ' $H e$ is' or 'She is"”). They were also instructed to type "he is" or "she is" at the beginning of every sentence.

\subsubsection{Customer Gapjil toward service employees}

After completing manipulation tasks, participants indicated their tendency of Gapjil which was operationalized as aggressiveness toward service employees. The aggressiveness scale from Reynolds and Harris (2009) was modified to be applicable in a service context. Participants read an instruction (i.e., "please think about service employees working at fast-food restaurants, gas stations, or convenient stores around you. To what extent do you agree with the statements below?") and indicated the extent of their agreement on six Gapjil-related sentences ("Given enough provocation, I may hit a service employee," "When service employees annoy me, I tell them what I think," "When frustrated by service employees, I let my irritation show," "Some of service employees that I met might think that I am hot-headed," "When service employees are especially nice, I wonder what they want," and "I sometimes find myself disagreeing with service employees," from $1=$ strongly disagree to $7=$ strongly agree, $\alpha=$ .81). The average score of six items was used as an index of Gapjil. Finally, demographic questions were given.

\subsection{Results and Discussion}

\subsubsection{Manipulation checks}

With respect to PEM, as expected, participants in the high PEM condition perceived higher economic mobility than those in the low PEM condition $\operatorname{did}\left(M_{\text {high }}=6.60, S D_{\text {high }}=2.29\right.$, $M_{\text {low }}=6.01, S D_{\text {low }}=2.08, t(229)=2.04, p=$ $.042, d=.27)$. In addition, regarding self-other referent priming, participants provided sentences in accordance with their assigned conditions.

\subsubsection{Hypothesis testing}

To test whether self-other referent priming moderates the effect of PEM on customer Gapjil, we regressed Gapjil on PEM $(-1=$ low PEM, 1 = high PEM), SORP $(-1=$ self, $1=$ other) and their interaction. Results revealed that main effects of PEM $(b=.05, t(227)=$ $.74, p=.460)$ and $\operatorname{SORP}(b=.06, t(227)=$ $.93, p=.354)$ were not significant. However, the interaction effect was significant $(b=$ -.13, $t(227)=-1.99, p=.048)$. More specifically, PEM marginally increased Gapjil in the self-referent priming condition ( $b=.18$, $t(227)=1.93, p=.055)$, whereas it did not affect Gapjil in the other-referent priming condition $(b=-.08, t(227)=-.88, p=$ 
.379). Therefore, results of study 1 supported hypothesis 1.

Findings of study 1 revealed that PEM increased Gapjil when people are self-referent primed, whereas PEM did not affect Gapjil when they are other-referent primed. Thus, study 1 showed that the effect of PEM on Gapjil varies depending on whether people give the relative attention to the self or others.

Our next reasonable question is whether this interaction effect of PEM and SORP on Gapjil always occurs. According to previous literature, temporal focus can affect self and other evaluation. Temporal focus refers to individuals' tendency of directing their attention to the past, the present, or the future (Shipp and Aeon 2019). Even though individuals characteristically focus on certain time periods, current cues influence the direction of momentary attention (Shipp and Aeon 2019). When people temporally focus on the present, we expect that PEM will increase Gapjil only when people are selfreferent primed (vs. other-referent primed), replicating the results of study 1 . Indeed, the manipulation of SORP in study 1 was to write sentences starting with either "I am" or "He/ she is." Because participants were asked to write the present-tense sentences, it is possible that they momentarily focused on the present. Conversely, when people temporally focus on

〈Figure 2〉 The Interaction Effect of PEM and Self-Other Referent Priming on Gapjil (Study 1)

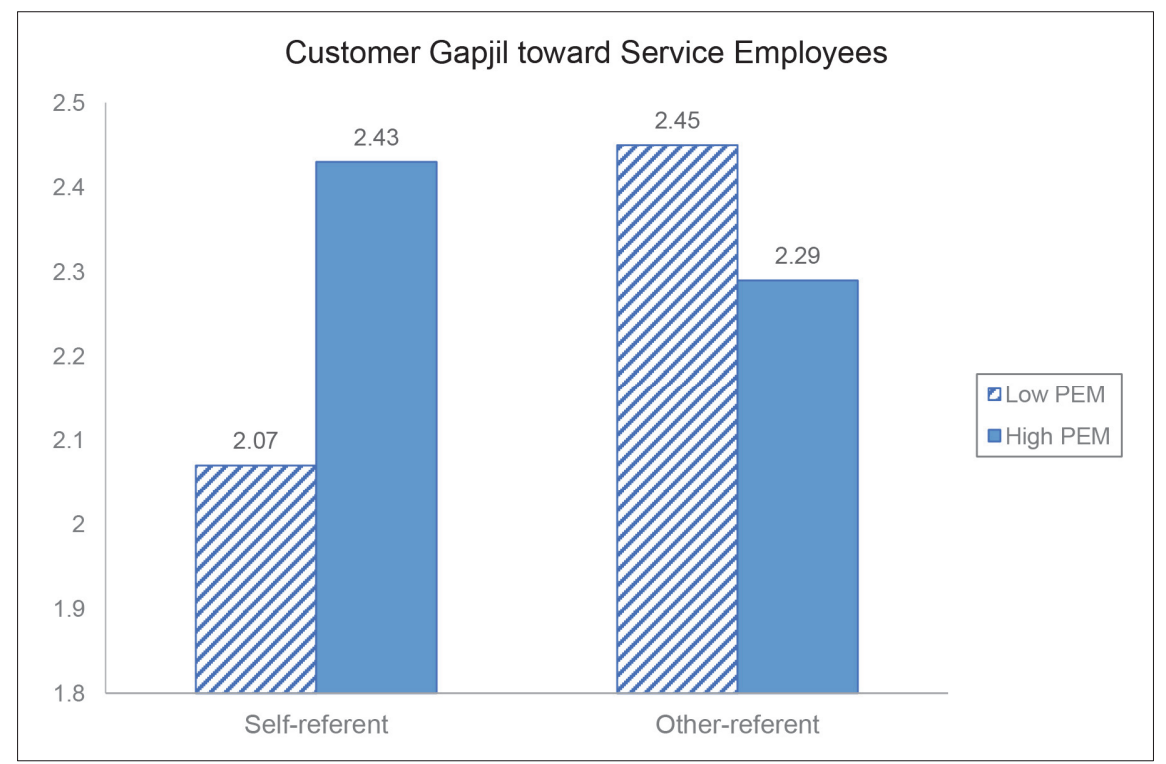

PEM $\times$ Self-other Referent Priming Effect: $b=-.13, t(227)=-1.99, p=.048$

In the self-referent priming condition, PEM effect: $b=.18, t(227)=1.93, p=.055$

In the other-referent priming condition, PEM effect: $b=-.08, t(227)=-.88, p=.379$ 
the future, PEM is expected to increase Gapjil more among other-referent primed people than among self-referent primed people. The reasoning behind this expectation is based on the effect of landmarks (Dai et al. 2015) and the better than average (BTA) improvement effect (Kanten and Teigen 2008).

People perceive temporal selves as a series of connected but distinct selves (Peetz and Wilson 2014). Perceived (dis)connection between the present self and a temporally removed self is malleable (Peetz and Wilson 2014). Previous studies have found that landmarks and transitions can affect the perceived connection between the present and other temporal selves (Bartels and Rips 2010; Peetz and Wilson 2013). We posit that people with high (vs. low) PEM are more likely to imagine the future self as accomplished, successfully employed, and financially affluent. Thus, people with high (vs. low) PEM might think of landmarks- or transitions-related financial achievements (e.g., promotion to an executive member or earning a high income) that fall between the present self and the future self. These landmarks might divide the current and future selves (Bartels and Rips 2010) and signal disconnection between temporal selves (Peetz and Wilson 2014). On the other hand, to people with low PEM, the future self might be similar to the current self. Therefore, when people focus on the future, people with high (vs. low) PEM are expected to perceive greater relative gratification, and adjust more their words and actions to the norm of the potential in-group (i.e., the rich).

Then, why do we expect the effect of PEM on Gapjil to be stronger in the other-referent (vs. self-referent priming) condition when people focus on future? Given ambiguity in forecasting the future, a motive for selfimprovement leads to higher improvement ratings for oneself than for others. That is, people believe that they would improve more than their peers in the future, and this effect is stronger in the distant future than in the near future (Kanten and Teigen 2008). When people think about the future, other-referent priming might enable people with high PEM to maximize the motive for self-improvement and to imagine a highly achieved self, compared with the present self and the future other. Therefore, in the future focus and other-referent priming condition, PEM would increase relative gratification and thus Gapjil. In the future focus and self-referent priming condition, the lack of other-related thoughts will not activate social comparison. Thus, the self-improvement motive might be weaker in this condition than in the future focus and other-referent priming condition. Therefore, we propose hypothesis 2 as follows.

Hypothesis 2: Temporal focus will moderate the interaction effect of PEM and SORP on customer Gapjil. 
Hypothesis 2A: Among people who focus on the present, PEM will increase Gapjil when people are self-referent primed, whereas PEM will not when people are other-referent primed.

Hypothesis 2B: Among people who focus on the future, PEM will increase Gapjil when people are other-referent primed, whereas PEM will not when people are self-referent primed.

\section{IN. Study 2: The Moderating Role of Temporal Focus}

Objectives of study 2 are twofold. First of all, the current study aims to test the moderating role of temporal focus (i.e., present vs. future) on the interactive effect of PEM and SORP on Gapjil. Second, the current study tests whether the three-way interaction effect is still sustained even when materialism, social dominance orientation, and optimism are controlled. Materialism and social dominance orientation are possibly related with customer Gapjil, and optimism is positively related with PEM (Yoon and Wong 2017). Thus, in an effort to test the robustness of findings, analysis included materialism, social dominance orientation, and optimism as covariates.

\subsection{Method}

A total of 241 participants were recruited from Amazon Mechanical Turk. One person who did not complete the study was excluded. Consequently, 240 participants (139 females, mean age $=39.18)$ were included in data analysis. Participants were composed of $84.6 \%$ Whites, $4.6 \%$ Asians, $4.2 \%$ African Americans, 3.3\% mixed ethnics, $0.8 \%$ American Indians or Alaska Natives, and $2.5 \%$ other. Study 2 employed a 2(SORP: self vs. other) x 2 (Temporal focus: present vs. future) betweensubjects design. PEM was measured in this study.

〈Figure 3〉 Research Framework

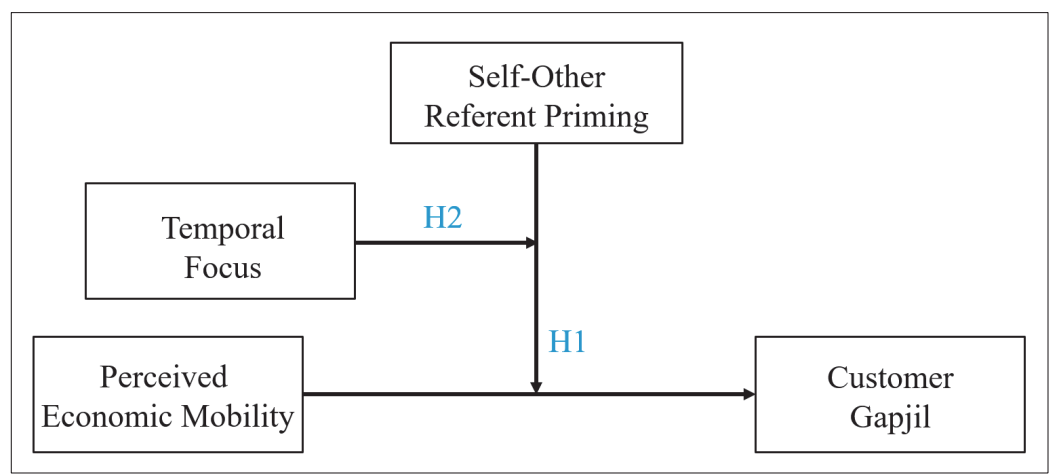

12 ASIA MARKETING JOURNAL Vol. 21 №. 04 January 2020 


\subsubsection{SORP}

Participants were randomly assigned to an either self- or other-referent priming condition. The same manipulation adopted from Wan et al. (2011) as in study 1 was used in this study, but participants provided twenty sentences in this study.

\subsubsection{Temporal focus}

After completing the self-other priming task, participants again were randomly assigned to an either present- or future-focus condition. Depending on the assigned condition, participants were asked to describe either daily lives at the present time (present-focus condition) or daily lives in the year of 2037 (future-focus condition). After the writing task, participants answered a temporal focus manipulation check question ("To what extent do you think that the day you have described above is temporally close to today?" from 1 = very close to $7=$ very distant).

\subsubsection{Customer Gapjil}

After completing manipulation-related tasks, participants indicated their tendency of Gapjil with the same items used in study 1 adapted from Reynolds and Harris (2009). The average score of the six items formed a Gapjil index $(a=.88)$.

\subsubsection{PEM}

PEM was measured by an eight-item scale adopted from Yoon and Wong (2017) (e.g., "Everyone has a fair chance at moving up the economic ladder"; "Starting in poverty does not put one at a distinct disadvantage in life"; $1=$ strongly disagree to 7 = strongly agree, $a=.95)$. The average score of the eight items formed a PEM index.

\subsubsection{Covariates}

The current study also included materialism adopted from Richins and Dawson (1992) (18 items, $a=.94)$, optimism from Scheier and Carver (1985) (8 items, $a=.92$ ), and social dominance orientation from Pratto et al. (1994) (16 items, $a=.96$ ). Although public self-consciousness was initially considered as a control variable, data analysis revealed that it did not affect Gapjil. Thus, this variable will not be discussed from now on.

\subsection{Results and Discussion}

\subsubsection{Manipulation checks}

In terms of temporal focus, as expected, participants in the future condition perceived the described day more distant from today than those in the present condition did ( $M_{\text {future }}$ $=4.71, S D=1.70, M_{\text {present }}=2.43, S D=1.61$, 
$t(238)=10.68, p<.001)$. As to self-other referent priming, participants wrote sentences consistent with their assigned conditions.

\subsubsection{Hypothesis testing}

To examine whether temporal focus moderates the interactive effect of PEM and SORP on Gapjil, we regressed Gapjil on mean-centered PEM, SORP ( -1 = self, 1 = other), temporal focus $(-1=$ present, $1=$ future $)$, and their all possible two-way and three-way interactions.
Among main effects, PEM significantly affected Gapjil $(b=.12, t(232)=2.02, p=.044)$, whereas SORP $(b=-.09, t(232)=-1.03$, $p=.303)$ and temporal focus $(b=-.03$, $t(232)=-.37, p=.709)$ did not have significant effects. None of two-way interaction effects was significant $(\mathrm{PEM} \times \mathrm{SORP}: \quad b=-.06$, $t(232)=-.96, p=.340 ; \mathrm{PEM} \times$ Temporal $:$ $b=.05, t(232)=.88, p=.379 ;$ SORP $\times$ Temporal: $b=.12, t(232)=1.40, p=.164)$. Most importantly, the three-way interaction effect of PEM, SORP, and temporal focus on

〈Figure 4〉

The 3-way Interaction Effect of PEM, Self-Other Referent Priming, and Temporal Focus on Gapjil (Study 2)

〈Figure 4-A〉 Present Focus (PEM is dichotomized for visual convenience.)

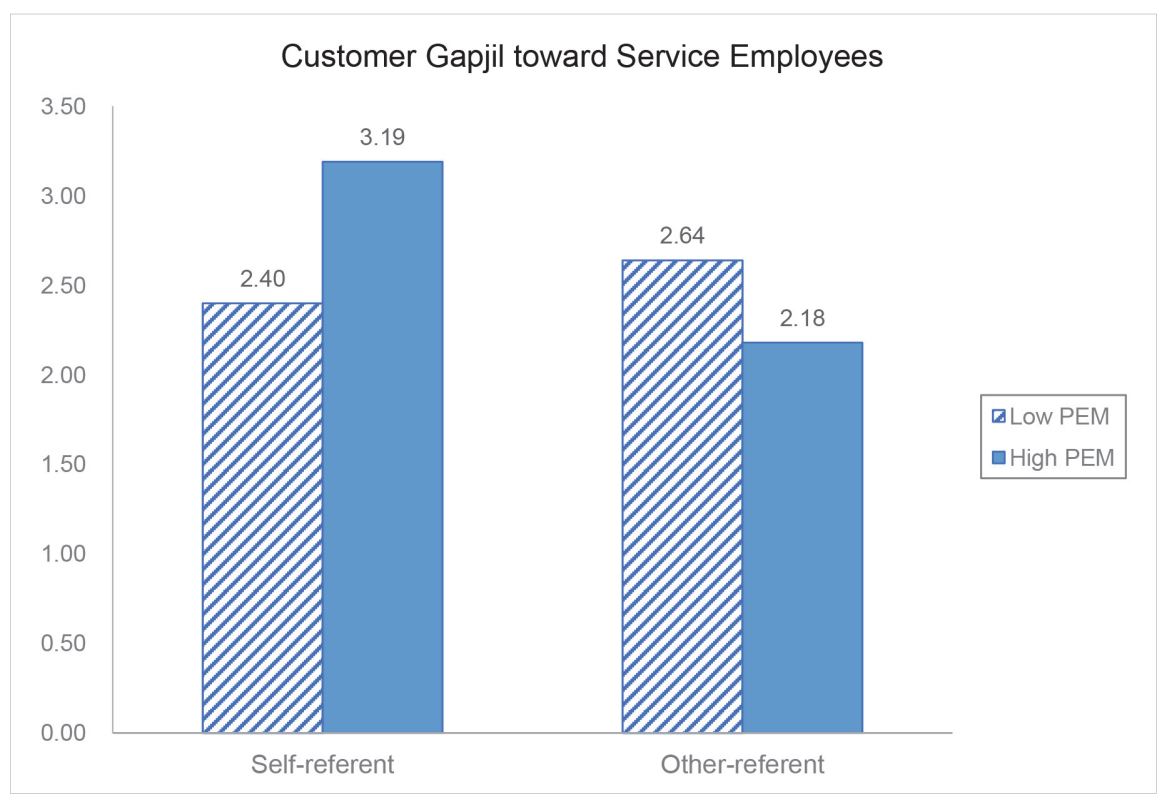

PEM $\times$ self-other interaction effect: $b=-.20, t(232)=2.36, p=.019$

In the self-referent priming condition, PEM effect: $b=.25, t(232)=2.26, p=.025$

In the other-referent priming condition, PEM effect: $b=-.15, t(232)=-1.16, p=.248$ 
〈Figure 4-B〉 Future Focus

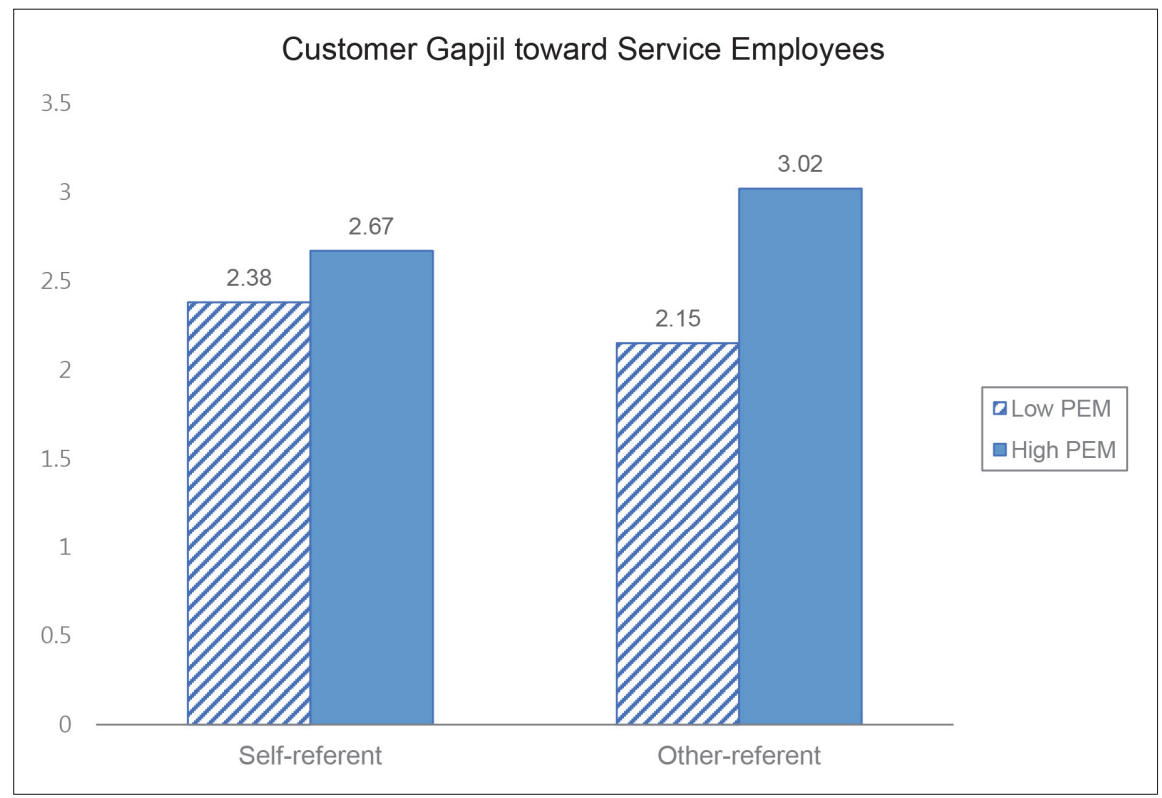

PEM $\times$ self-other interaction effect: $b=.10, t(232)=1.10, p=.272$

In the self-referent priming condition, PEM effect: $b=.09, t(232)=.76, p=.450$

In the other-referent priming condition, PEM effect: $b=.28, t(232)=2.28, p=.024$

Gapjil was significant $(b=.15, t(232)=$ 2.44, $p=.015)$. Specifically, in the present focus condition, the two-way interaction effect of PEM and SORP was significant $(b=-.20$, $t(232)=2.36, p=.019)$. A further investigation revealed results consistent with hypothesis $2 \mathrm{~A}$ such that PEM increased Gapjil in the selfpriming condition $(b=.25, t(232)=2.26, p$ $=.025)$, whereas PEM did not affect Gapjil in the other-priming condition $(b=-.15, t(232)$ $=-1.16, p=.248)$. Conversely, in the future focus condition, the two-way interaction effect of PEM and self-other priming was not significant $(b=.10, t(232)=1.10, p=$ .272). Simple effect analyses revealed results consistent with hypothesis 2B; PEM increased Gapjil in the other-priming condition $(b=.28$, $t(232)=2.28, p=.024)$, whereas PEM did not in the self-priming condition $(b=.09$, $t(232)=.76, p=.450)$. Overall, results of study 2 supported hypotheses $2 \mathrm{~A}$ and $2 \mathrm{~B}$.

We also tested whether the three-way interaction effect is sustained even when materialism, social dominance orientation, and optimism are controlled. In this analysis, Gapjil was regressed on mean-centered PEM, SORP, temporal focus, their all possible interactions, and covariates (i.e., materialism, social dominance orientation, and optimism). All covariates significantly influenced Gapjil in expected ways: 
materialism $(b=.26, t(229)=2.57, p<$ .001 ) and social dominance orientation ( $b=$ $.25, t(229)=4.12, p<.001)$ increased Gapjil, whereas optimism $(b=-.17, t(229)=-2.56$, $p=.011$ ) decreased Gapjil. Most importantly, the three-way interaction effect of PEM, SORP, and temporal focus was still significant $(b=.14, t(229)=2.57, p=.011)$ even when aforementioned covariates were controlled. Thus, the three-way interaction effect was robust.

In summary, study 2 tested the moderating role of temporal focus on the interaction effect of PEM and SORP on Gapjil, supporting hypotheses $2 \mathrm{~A}$ and $2 \mathrm{~B}$. When people focused on the present, PEM induced Gapjil when they thought of self rather than others. On the other hand, when people focused on the future, PEM increased Gapjil when they thought of others rather than self.

\section{General Discussion}

The current research investigated the intertwined effects of PEM, SORP, and temporal focus on customer Gapjil toward service employees. We theorized that PEM, SORP, and temporal focus jointly affect the degree of perceived relative gratification which motivates a customer to attune the self to the perceived norm of the high class, influencing Gapjil. The results of two studies were consistent with our theorizing. In study 1, PEM increased Gapjil among selfreferent primed people, whereas it did not change Gapjil among other-referent primed people. In study 2, the interaction effect of PEM and SORP on Gapjil was moderated by temporal focus. In the present-focus condition, PEM increased Gapjil only among people who thought of self but not among those who thought of others, replicating the results of study 1. However, in the future-focus condition, PEM increased Gapjil among other-referent primed people, but not among self-referent primed people, even though the direction was equal.

\subsection{Theoretical Contributions}

As previously mentioned, customers are often considered as a group of people conducting Gapjil toward service employees and companies, and customer Gapjil decreases physical and emotional well-being of service employees and financial performance of companies. Despite the increased attention to customer Gapjil in reality, academic endeavors to delve into this phenomenon has lagged behind in the marketing field. The present research made the first attempt to empirically test what brings the phenomenon.

The current research focused on three factors, PEM, SORP, and temporal focus, as antecedents of customer Gapjil. PEM reflects people's lay theories in reality such as "Spoon Hierarchical 
Theory" in Korea and "American dreams" in the U.S. We viewed PEM as an important concept in investigating Gapjil in that the perception might affect one's expected socioeconomic status in the future and thus might influence how to attune one's words and actions in interpersonal relationships. Although previous studies regarding PEM have found its socially desirable consequences in contexts of well-being and financial behaviors, quite surprisingly, Kwon and Yi (2019) found that PEM increases customer aggression toward service employees.

The current research extends their finding by adding two important dimensions - the intra-personal vs. inter-personal focus (i.e., SORP) and the present vs. future focus (i.e., temporal focus). By showing that the effect of PEM on Gapjil dynamically varies depending on the relative attention given to each of these dimensions, the current research provides a theoretical framework that is helpful in predicting customer Gapjil based on psychological states and focuses. In addition, the current research provides conceptual explanations about the 3-way interaction effect. We conceptualize the moderating effect of SORP on the relationship between PEM and Gapjil with self- and otherreferent knowledge used in social comparisons. We also theorize the moderating effect of temporal focus with perceptions of distinctive selves, especially among people with high PEM, between before and after expected financial achievements (i.e., landmarks) and with the better than average improvement effect.

We expect that this initial attempt to empirically test the antecedents could facilitate future research on customer Gapjil. Additional efforts to establish the nomological network of Gapjil could invite numerous theories in marketing, social psychology, and other relevant fields which might deepen our understanding of Gapjil. The theoretical network could enable marketing researchers to interpret and predict this detrimental phenomenon from a psychological perspective. The current research provides theoretical contributions to academia by laying the foundation stone of the nomological network of Gapjil.

\subsection{Practical Implications}

By manipulating PEM (in study 1), SORP (in study 1 and 2), and temporal focus (in study 2), the current research showed that each of these antecedents can be momentarily modified. Accordingly, it has practical implications for communication strategies (e.g., advertising concepts, media slogans, or public agenda) of companies and governments to lessen Gapjil in our society. However, one important question should be answered before the institutions take actions: is high PEM always a bad thing that leads to Gapjil? We would not say yes. We argued that PEM increases relative gratification which motivates people to attune themselves 
to the perceived norm of the high status. In turn, PEM induces Gapjil.

What if people perceive the norm of the high class in a different way? If ordinary people directly or indirectly experienced high status role models who act according to the philosophy of noblesse oblige, the perceived norm of the high status would be modesty, consideration, and responsibility. Thus, it would be helpful in decreasing Gapjil to expose exemplars of the high class who conduct noblesse oblige and are respected within the society. This societal endeavor might change the direction of PEM's effect on Gapjil. Under such a social atmosphere, people with high PEM might focus on those norms of the higher class and attune themselves to the norms. Based on the results of this first remedy, one could further adjust SORP and temporal focus to weaken Gapjil in our society. Under this circumstance, people with high PEM might conduct Gapjil less than those with low PEM when they are self-focus primed, showing the reversed effect found in our study 1. The effect of temporal focus might also be reversed under societies led by the philosophy of noblesse oblige. To conclude, the perceived norm of the high class in a society seemingly has a critical role in determining the direction of the intertwined effects of PEM, SORP, and temporal focus on Gapjil. Depending on the norm of the high class in each of societies, governments or service companies need to have different communication strategies in terms of adjusting SORP and temporal focus to create a "Gapjil-free" society.

In addition, we also recognize the perils of a slogan advocating "the customer is the king," which can aggravate Gapjil. This type of slogan can produce a social atmosphere under which a customer acts like a king, and a service employee acts like a servant. Particularly, customers of high-end services might think that they have the right to have a service employee at their beck, because they spend a large amount of money on the service. People with high PEM might dream of using service workers like their servants, as the aforementioned slogan states them as "a king." Overall, "a customer as a king" slogan might create a social pressure which forces service employees to adapt themselves to customer Gapjil without any resistance in order to maintain their job positions (Choi 2018). Therefore, as a communication strategy, it could be desirable to present slogans highlighting the equal relationship between customers and employees in establishing a "Gapjil-free" society. These communicational efforts could prevent people from considering service employees or emotional laborers as inferior beings.

\subsection{Limitations and Future Research}

There is still room for future research. First of all, the current research used aggressiveness toward service employees as an operationalization 
of customer Gapjil. This operationalization captures customers' intention to use aggressive words and actions in the interpersonal relationship between customers and employees. However, there could be other types of customer Gapjil. For example, some customers might not show aggressiveness to service employees but spread bad word of mouth about the service company on SNS. Meanwhile, other customers might kindly ask an unreasonable favor such as returning electronic products broken by their fault, claiming that the product was already broken when first unpacked. Thus, further works on classifying types and developing a sophisticated scale of Gapjil will facilitate research on this important phenomenon.

In addition, customers may experience Gapjil in various contexts. Customers might witness Gapjil of a manager toward a junior employee in a store or Gapjil of other customers who have a higher status in the service context or promotion programs toward customers (e.g., from a passenger in the business class to one in the economy class) or service workers (e.g., from a passenger in the business class to a flight attendant). Therefore, future research needs to investigate not only Gapjil conducted by customers but also Gapjil by other agents which might have an influence on consumer behaviors.

Finally, the effect of temporal focus might be different depending on the history of countries. For example, Chinese culture emphasizes traditions and ancestors in its long history. In this culture, Chinese citizens focus more on the past, compared with North Americans (Hofstede et al. 2005). Thus, one could test the findings of this research in various cultures with different levels of history in order to extend our understanding of how chronic and momentary temporal focuses affect customer behavior.

In conclusion, the present research tries to figure out causal factors inducing customer Gapjil. Given that customer Gapjil decreases well-being of millions of service workers and financial performance of many service companies and stores, the current research highlights the importance of finding causal factors in order to lessen Gapjil in our society. We hope that the current endeavor facilitates future research on the phenomenon in marketing.

〈Received September 4. 2019〉

$\langle$ Accepted January 6. 2020〉

\section{References}

Bartels, Daniel M. and Lance J. Rips (2010), "Psychological Connectedness and Intertemporal Choice," Journal of Experimental Psychology: General, 139(1), 49-69.

Cho, Sun-Bai (2018), "Jaycustomers and KapzilCulture in Korean Society," Korean Journal of Hospitality \& Tourism, 27(5), 207-22. Choi, Hangsub (2018), "Sociological Understanding 
of 'Gapjil' of All the Classes in the Korean Society," Social Science Studies, 30 (2), 225-48.

Dai, Hengchen, Katherine L. Milkman, and Jason Riis (2015), "Put Your Imperfections Behind You: Temporal Landmarks Spur Goal Initiation When They Signal New Beginnings," Psychological Science, 26(12), 1927-36.

Dambrun, Michaël, Donald M. Taylor, David A. McDonald, Jonathan Crush, and Alain Me'ot (2006), "The Relative DeprivationGratification Continuum and the Attitudes of South Africans toward Immigrants: A Test of the V-Curve Hypothesis," Journal of Personality and Social Psychology, 91 (6), 1032-44.

Enosh, Guy, Shay Tzafrir, and Amit BenAhron Gur (2013), "Client Aggression toward Social Workers and Social Services in Israel - A Qualitative Analysis," Journal of Interpersonal Violence, 28(6), $1123-42$.

Fenigstein, Allan and Michael P. Levine (1984), "Self-Attention, Concept Activation, and the Causal Self," Journal of Experimental Social Psychology, 20(3), 231-45.

Förster, Jens, Nira Liberman, and Ronald S. Friedman (2007), "Seven Principles of Goal Activation: A Systematic Approach to Distinguishing Goal Priming from Priming of Non-Goal Constructs," Personality and Social Psychology Review, 11(3), 211-33.
Gong, Taeshik (2015), “Internal Service Recovery's Influence on Frontline Service Employees' Satisfaction and Loyalty," Asia Marketing Journal, 17(2), 39-62.

Guimond, Serge and Michaël Dambrun (2002), "When Prosperity Breeds Intergroup Hostility: The Effects of Relative Deprivation and Relative Gratification on Prejudice," Personality and Social Psychology Bulletin, 28(7), 900-12.

Han, Youngjun (2018), “No.1 Key Success Factor? 'Parental Wealth',' Financial News. Hofstede, Geert, Gert Jan Hofstede, and Michael Minkov (2005), Cultures and Organizations: Software of the Mind. New York: Mcgraw-hill.

Jeon, In-Soo and Jae-Min You (2014), “The Management of Contact Person as Emotional Labor: Based on Self-Determination Theory and Customer Orientation," Korean Journal of Marketing, 29(5), 77-99.

Jeong, Han-Wool and Gye-Won Jo (2019), "An Empirical Study on 'Gapjil' Culture in Korean Society," Korean Political Science Review, 53(1), 105-25.

Kanten, Alf Børre and Karl Halvor Teigen (2008), "Better Than Average and Better With Time: Relative Evaluations of Self and Others in the Past, Present, and Future," European Journal of Social Psychology, 38(2), 343-53.

Kim, Haeyoen (2016.12.15), “Endemic Gapjil Everywhere... Special Crackdown Arrested 
7,000 People," Yonhap News.

Krueger, Joachim and David Stanke (2001), "The Role of Self-Referent and OtherReferent Knowledge in Perceptions of Group Characteristics," Personality and Social Psychology Bulletin, 27(7), 878-88. Kwon, Yongju and Youjae Yi (2019), “The Effect of Perceived Economic Mobility on Customer Aggression toward Service Employees: A Darker Aspect of Customer Behavior," Psychology and Marketing, 36 (11), 1120-32.

Lee, Jae-Ho (2016a), "The Superordinate's Abuse of Power in Korean Society: Sociocultural and Psychological Viewpoints," Pastoral Care and Counseling, 27, 172-202. Lee, Sarah (2016b), "Pathological Personality and the Society: Focus on the Narcissistic Personality Disorder," Journal of Modern Social Science, 20, 31-47.

Moon, Younhee (2018), "Customer-Contact Employee Support and Service Recovery Efforts : The Mediating Role of Job Burnout and Customer Orientation," Asia Marketing Journal, 20(3), 83-103.

Oppenheimer, Daniel M., Tom Meyvis, and Nicolas Davidenko (2009), "Instructional Manipulation Checks: Detecting Satisficing to Increase Statistical Power, Journal of Experimental Social Psychology, 45(4), 867-72.

Park, In-Ho (2016), "The Protection for Emotional Laborers and the Responsibilities of Businesses Thereto," Kookmin Law Review, 36(1), 927-55.

Peetz, Johanna and Anne E. Wilson (2014), "Marking Time: Selective Use of Temporal Landmarks as Barriers between Current and Future Selves," Personality and Social Psychology Bulletin, 40(1), 44-56. (2013), "The Post-Birthday World: Consequences of Temporal Landmarks for Temporal Self-Appraisal and Motivation," Journal of Personality and Social Psychology, 104(2), 249.

Postmes, Tom and Laura G. E. Smith (2009), "Why Do the Privileged Resort to Oppression? A Look at Some Intragroup Factors," Journal of Social Issues, 65(4), 769-90.

Pratto, Felicia, Jim Sidanius, Lisa M Stallworth, and Bertram F Malle (1994), "Social Dominance Orientation: A Personality Variable Predicting Social and Political Attitudes," Journal of Personality, 67(4), 741-63.

Rafaeli, Anat, Amir Erez, Shy Ravid, Rellie Derfler-Rozin, Dorit Efrat Treister, and Ravit Scheyer (2012), “When Customers Exhibit Verbal Aggression, Employees Pay Cognitive Costs," Journal of Applied Psychology, 97(5), 931-50.

Reynolds, Kate L. and Lloyd C. Harris (2009), "Dysfunctional Customer Behavior Severity: An Empirical Examination," Journal of Retailing, 85(3), 321-35. 
Richins, Marsha L. and Scott Dawson (1992), “A Consumer Values Orientation for Materialism and Its Measurement: Scale Development and Validation," Journal of Consumer Research, 19(3), 303-16.

Ryu, Hyung-Seo and Ki-Sang Ryu (2017), 'Influence of Customer's 'GABJIL' on Employee's Emotional Burnout, Counterproductive Work Behavior and Turnover Intention in the Foodservice Industry," Korean Journal of Hospitality \& Tourism, 26(7), 39-54.

Scheier, Michael F. and Charles S Carver (1985), "Optimism, Coping, and Health: Assessment and Implications of Generalized Outcome Expectancies," Health Psychology, 4(3), 219-47.

Seo, Min (2015), "Memories of Gapjil," Inmul kwa Sasang, 6-9.

Shipp, Abbie J. and Brad Aeon (2019), "Temporal Focus: Thinking about the Past, Present, and Future," Current Opinion in Psychology, 26, 37-43.

Verbeke, Willem and Richard P. Bagozzi (2002), "A Situational Analysis on How Salespeople Experience and Cope with Shame and Embarrassment," Psychology and Marketing, 19(9), 713-41.
Wan, Lisa C., Michael K. Hui, and Robert S. Wyer (2011), "The Role of Relationship Norms in Responses to Service Failures," Journal of Consumer Research, 38(2), 260-77.

Wood, Noelle and Nelson Cowan (1995), "The Cocktail Party Phenomenon Revisited: How Frequent Are Attention Shifts to One's Name in an Irrelevant Auditory Channel?," Journal of Experimental Psychology: Learning, Memory, Cognition, 21 (1), 255-60.

Yi, Youjae (2014), Customer Value Creation Behavior, NY: Routledge.

Yoon, Hae-sung, Jung-ju Lee, Jong-seol Yoon, Yong-hwan Joo, and Sun-joong Lee (2018), "A Study of the Causes and Comprehensive Measures to Tackle 'Gapjil' within the Public Sector," Korean Institute of Criminology. Yoon, Sunyee and Hyeongmin Christian Kim (2016), "Keeping the American Dream Alive: The Interactive Effect of Perceived Economic Mobility and Materialism on Impulsive Spending," Journal of Marketing Research, 53(5), 759-72.

Yoon, Sunyee and Nancy Wong (2017), "Understanding Individual Differences in Perceived Economic Mobility," Working Paper. 


\title{
FINANCIAL TIMES
}

\section{British Dream: Good news on economic mobility}

\author{
By JASON DEPARLE
}

LONDON - Joanne Rowling did it. Thomas Hunter did it. And British life is built on the faith that others can do it, too: rise from humble origins to economic heights. "Movin' on up" is a civil religion.

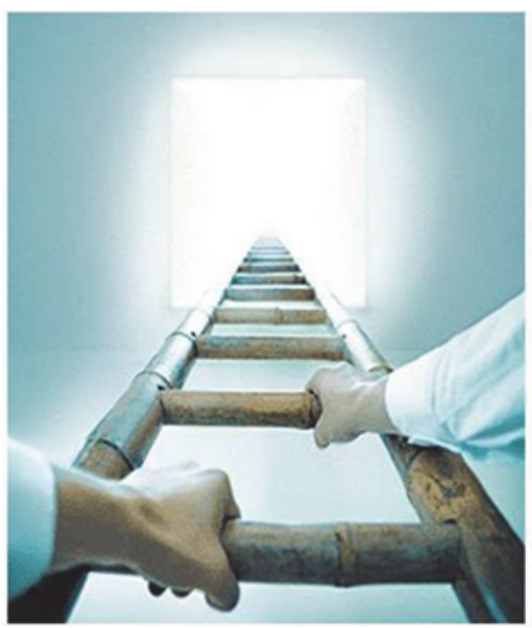

Despite increasing concern about it, many researchers have reached a conclusion that confirms conventional wisdom: the United Kingdom is still the land of opportunity.

Economists spent the last decade tracing the impact of parental income on child outcomes. At least five large studies in recent years have found the United Kingdom to be more mobile than comparable nations in Europe, as well as Australia and Canada.

Indeed, a project led by Markus Forsberg, an economist at a Swedish university, found that 19 percent of British men raised in the bottom fifth of incomes rose to the top fifth. Meanwhile, just 11 percent of British men at the bottom stay there as adults. Anyone born to a poor or less-educated family has a chance of escaping poverty and climbing the income ladder.

"Children in the U.K. do not necessarily have to end up in the same place as their parents," Isabel V. Sawhill, an economist at the National Institute of Economic and Social Research (NIESR), said in an interview. Where you start does not decide where you finish. The most guaranteed way to succeed in the United Kingdom is to work hard and build your own skills.

5 - 2 o save e e-mall $\mathbf{0}$ share 


\section{FINANCIAL TIMES}

British Nightmare: What happened to economic mobility?

\section{By JASON DePARLE}

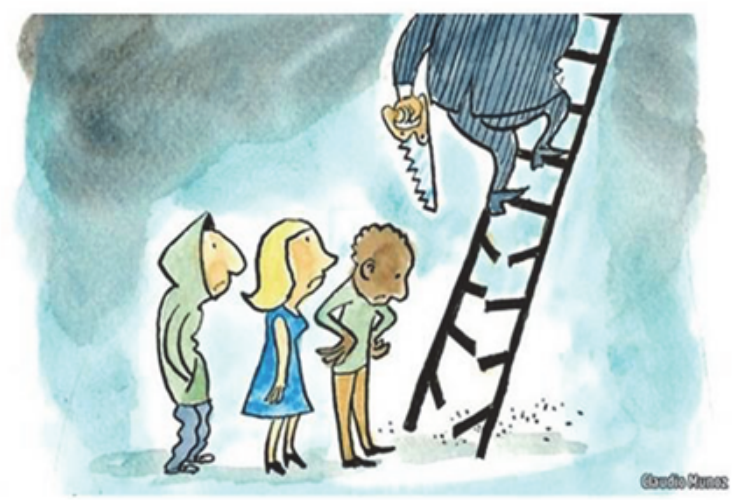

LONDON - Joanne Rowling did it. Thomas Hunter did it. And British life is built on the faith that others can do it, too: rise from humble origins to economic heights.

But many researchers have reached a conclusion that turns conventional wisdom on its head: the United Kingdom is no longer the land of opportunity that it once was.

Economists spent the last decade tracing the impact of parental income on child outcomes. At least five large studies in recent years have found the United Kingdom to be less mobile than comparable nations in Europe, as well as Australia and Canada.

Indeed, there is growing evidence of a strong link between parental income and children's economic outcomes. A project led by Markus Forsberg, an economist at a Swedish university, found that 67 percent of British men raised in the bottom fifth of incomes stay there as adults. Meanwhile, just 4 percent of British men at the bottom rose to the top fifth.

"Children in the U.K. are much more likely as adults to end up in the same place on the income ladder as their parents," Isabel V. Sawhill, an economist at the National Institute of Economic and Social Research (NIESR), said in an interview. The most guaranteed way to succeed in the U.K. is to born with a silver spoon in your mouth. Life is a game, but it's NOT fair.

I) 20 D save a email $\mathbf{t}$ share 